\title{
Efeitos de períodos de permanência do flumioxazin no solo e na palha de milho e aveia na eficácia de controle de plantas daninhas
}

\author{
Effects of flumioxazin permanence period in soil and in corn and oat mulch on \\ weed control efficacy
}

\author{
Caio Antonio Carbonari ${ }^{1}$, Giovanna Larissa Gimenes Cotrick Gomes ${ }^{2}$, \\ Edivaldo Domingues Velini ${ }^{3}$
}

\begin{abstract}
Resumo- O presente trabalho teve por objetivo avaliar a eficácia de flumioxazin aplicado sobre o solo e sobre a palha de aveia e milho no controle de plantas daninhas, após diferentes períodos sem chuvas. $\mathrm{O}$ experimento foi conduzido em vasos, em condições de casa de vegetação, no município de Botucatu-SP. Os vasos foram preenchidos com solo, e as sementes de plantas daninhas (Brachiaria decumbens, Ipomoea nil, Digitaria spp, Bidens pilosa, Ipomoea grandifolia e Sida rhombifolia) foram semeadas e, em seguida, cobertas ou não com palha de aveia e milho, dependendo do tratamento utilizado. Para cada espécie de cobertura (aveia e milho), os tratamentos foram constituídos em esquema fatorial $2 \times 4$, em delineamento inteiramente casualizado, com quatro repetições, sendo dois posicionamentos do herbicida, (sobre o solo e sobre a palha) e quatro períodos sem ocorrência de chuvas após a aplicação do herbicida $(1,15$, 30 e 60 dias), além de testemunhas sem aplicação do produto, na presença e ausência de palha. De maneira geral, observou-se que a aplicação de flumioxazin sobre a palha de milho e aveia promoveu excelentes níveis de controle das plantas daninhas. Também houve tendência de redução nos níveis de controle para algumas espécies de plantas daninhas quando o produto foi exposto a períodos superiores à 30 dias entre a aplicação e a ocorrência de chuvas.
\end{abstract}

Palavras-chave: Cobertura morta, plantio direto, herbicida.

\begin{abstract}
This work aimed to evaluate the efficacy of flumioxazin applied over soil and over oat and corn mulch for controlling weeds, after different periods of permanence without rain. The experiment was carried out in vases, under greenhouse conditions in Botucatu County, São Paulo, Brazil. Vases were filled with soil, and weeds seeds of Brachiaria decumbens, Ipomoea nil, Digitaria spp, Bidens pilosa, Ipomoea grandifolia and Sida rhombifolia were sown and covered or not with oat and corn mulch, depending on the treatment used. For each coverage specie (oat and corn), treatments were constituted by factorial scheme $2 \times 4$, in randomized entirely design, with four repetitions, with two positions of herbicide application (over soil and over mulch) and four periods without occurrence of rain after herbicide application (1, 15, 30, and 60 days), besides checks without product application, in presence or absence of mulch. It was observed that

\footnotetext{
$1_{*}$ Eng. Agr. Dr., Depto. de Produção Vegetal, FCA/UNESP, Fazenda Experimental Lageado s/n, Caixa Postal 237, 18603-970, Botucatu-SP, e-mail: carbonari@fca.unesp.br

${ }^{2}$ Mestranda em Agronomia, Depto. de Produção Vegetal, FCA/UNESP, 18603-970, Botucatu-SP.

${ }^{3}$ Prof. Dr. Livre-Doente, Depto. de Produção Vegetal, FCA/UNESP, 18603-970, Botucatu-SP.
} 
flumioxazin application over corn and oat mulch promoted excellent weeds control levels. It was also observed a tendency of reduced control levels for some weeds species when the product was exposed for periods longer than 30 days between the application and the occurrence of rains.

Keywords: Mulch, soybean, no-tillage, herbicide

\section{Introdução}

O sistema de plantio direto da soja sobre a palha é uma técnica de manejo que está consolidada em várias regiões brasileiras. Os três princípios básicos do sistema de plantio direto são a cobertura permanente, o não revolvimento do solo e a rotação de culturas.

Os restos culturais deixados na superfície do solo após a colheita são consequiência da rotação de culturas, em sistema de plantio direto, a qual desempenha papel importante no sistema plantio direto, pois além de incrementar o teor de matéria orgânica do solo, proporcionando ao mesmo benefícios nas suas propriedades físicoquímicas e biológicas (Miyazawa et al., 2000), pode ser uma barreira à germinação e emergência de plantas daninhas (Gomes Jr \& Christoffoleti, 2008).

Esse efeito depende, por sua vez, do tipo de cobertura proporcionada pela massa vegetal, da distribuição e da quantidade. Entre as características desejáveis das espécies de cobertura destaca-se o suprimento de nutrientes com a decomposição da palha e a supressão de plantas daninhas, pelo efeito físico ou alelopático (Moraes et al., 2009).

Para a formação de palha, as principais culturas que compõe boas sucessões com a soja são a aveia, o milheto e o sorgo; além do milho-safrinha, o girassol e o trigo, que são utilizados também para a produção de grãos, oferecendo uma segunda fonte de renda ao produtor (Fidelis et al., 2003).
Para o estabelecimento do plantio direto são necessários cuidados específicos de gerenciamento, dentre os quais se destaca o eficiente controle das plantas daninhas (Gomes Jr \& Christoffoleti, 2008). O manejo integrado de plantas daninhas é essencial para o controle eficiente das mesmas em plantio direto, utilizando-se todos os métodos de controle possíveis, o que contribui para um manejo eficiente e economicamente viável para manter a sustentabilidade do sistema (Paes \& Rezende, 2001).

Flumioxazin é um herbicida registrado para uso em condições de pré-emergência, no controle de plantas daninhas de folhas largas e de algumas monocotiledôneas na cultura da soja (Oliveira et al., 1998); apresenta baixa solubilidade em água $\left(1,79 \mathrm{mg} \mathrm{L}^{-1}\right.$ a $\left.25^{\circ} \mathrm{C}\right) \mathrm{e}$ pressão de vapor $\left(2,41 \times 10^{-6} \mathrm{mmHg}\right.$ a $\left.22^{\circ} \mathrm{C}\right)$, o que sugere que ele tenha baixo potencial de volatilização (Rodrigues \& Almeida, 2005).

A presença de resíduos vegetal na superfície do solo após a dessecação, em présemeadura, pode diminuir a ação dos herbicidas aplicados. Isso se deve à interceptação das gotas antes que elas cheguem até o solo, atribuída principalmente à retenção e/ou possibilidade de degradação e volatilização do herbicida interceptado (Locke \& Bryson, 1997), constituindo-se uma das dificuldades do manejo de plantas daninhas em áreas de plantio direto. A retenção dos herbicidas pela palhada pode ser atribuída às diferentes características físicoquímicas dos produtos, às quantidades e origem da cobertura morta e à intensidade e época de ocorrência de chuvas após a aplicação dos produtos (Rodrigues, 1993; 
Fornarolli et al., 1998; Rossi, 2004; Maciel \& Velini, 2005; Tofoli et al., 2009), a qual participa decisivamente na capacidade de um herbicida aplicado em pós-emergência atingir o solo e atuar no controle de plantas daninhas (Carbonari et al., 2010).

Para o herbicida flumioxazin são escassas na literatura informações sobre o seu comportamento quando aplicados sobre a palha. Diante do exposto, o objetivo do trabalho foi avaliar os efeitos de diferentes períodos de permanência do herbicida flumioxazin na palha de aveia, milho e no solo, na sua eficácia de controle de plantas daninhas.

\section{Material e Métodos}

Dois experimentos foram conduzidos em casa de vegetação, no ano de 2010, no Núcleo de Pesquisas Avançadas em Matologia (NUPAM), pertencente à Faculdade de Ciências Agronômicas (FCA/UNESP) no município de Botucatu. Foram utilizados vasos com capacidade para 2 litros de solo, sendo utilizado como substrato, um Latossolo Vermelho Escuro (LVd), com as seguintes características químicas, $\mathrm{pH}\left(\mathrm{CaCl}_{2}\right),=4,3$; M.O. $\left(\mathrm{g} / \mathrm{dm}^{3}\right)=$ 19 ; SB e CTC $=14,6$ e 73 e físicas eram: $76 \%$ de areia, $20 \%$ de argila e $4 \%$ de silte, sendo classificado como de textura média. Realizou-se uma adubação de correção do solo para que as condições de fertilidade fossem melhoradas e proporcionasse uma melhor manutenção e desenvolvimento das plantas daninhas.

Foram semeadas seis espécies de plantas daninhas Brachiaria decumbens, Ipomoea nil; Digitaria sp., Bidens pilosa; Ipomoea grandifolia e Sida rhombifolia. Para todas as espécies foram adicionadas quantidades de sementes nos vasos para que se obtivessem 25 plantas de cada espécie por vaso. A quantidade de palha de aveia e de milho sobre o solo, utilizada para cada vaso foi calculada para representar 6 toneladas de massa seca por hectare.

Para cada espécie de cobertura (aveia e milho), os tratamentos foram constituídos em um esquema fatorial $2 \times 4$, em delineamento inteiramente casualizado (4 repetições), sendo dois posicionamentos do herbicida, (sobre o solo e sobre a palha) e quatro períodos sem ocorrência de chuvas após a aplicação do herbicida (1, 15, 30 e 60 dias), além de testemunhas sem aplicação do produto, na presença e ausência de palha. A quantidade de chuva simulada em cada tratamento foi de 20 $\mathrm{mm}$.

A aplicação do herbicida e a simulação de chuvas $(20 \mathrm{~mm})$ foram realizadas através de um equipamento instalado em laboratório. $\mathrm{O}$ flumioxazin (Flumyzin) foi aplicado na dose de $60 \mathrm{~g}$ i.a. ha ${ }^{-1}$ através de um pulverizador equipado com uma barra contendo quatro pontas XR11002 e foi operado sobre pressão constante de 1,5 bar, pressurizado por ar comprimido, com consumo de calda de $200 \mathrm{~L}$ $\mathrm{ha}^{-1}$.

As avaliações de controle foram realizadas aos 14, 28 e 42 dias após a simulação da chuva (DAC), por notas visuais, segundo escala percentual de notas, onde "0"corresponde a nenhum controle e "100" corresponde ao controle total das plantas daninhas (SBCPD, 1995).

Os tratamentos foram dispostos em um delineamento inteiramente casualizado, com quatro repetições. Os resultados obtidos foram submetidos à análise de variância pelo teste "F" a 5\% de probabilidade e as médias foram comparadas pelo teste Tukey a $5 \%$ de probabilidade.

\section{Resultados e discussão}

Nas Tabelas 1, 2 e 3 estão apresentados os resultados de controle das plantas daninhas pelo flumioxazin aplicado no solo e sobre a palha de milho. Para B. decumbens foram 
observados excelentes resultados de controle aplicação sobre a palha, para os diferentes (acima de 90\%) para a aplicação sem palha e períodos estudados.

bons níveis de controle (acima de $80 \%$ ) para a

Tabela 1. Eficácia do flumioxazin no controle de Brachiaria decumbens e Digitaria sp. aplicado no solo e na palha de milho, após diferentes períodos sem chuvas. Botucatu/SP 2010.

\begin{tabular}{|c|c|c|c|c|c|c|}
\hline \multirow[b]{3}{*}{ Períodos (dias) } & \multicolumn{6}{|c|}{ Intervalos de avaliação } \\
\hline & \multicolumn{2}{|c|}{14 DAC } & \multicolumn{2}{|c|}{28 DAC } & \multicolumn{2}{|c|}{42 DAC } \\
\hline & $\begin{array}{l}\text { Com } \\
\text { palha }\end{array}$ & $\begin{array}{c}\text { Sem } \\
\text { palha } \\
\end{array}$ & $\begin{array}{c}\text { Com } \\
\text { palha } \\
\end{array}$ & $\begin{array}{c}\text { Sem } \\
\text { palha }\end{array}$ & $\begin{array}{c}\text { Com } \\
\text { palha }\end{array}$ & $\begin{array}{c}\text { Sem } \\
\text { palha }\end{array}$ \\
\hline & \multicolumn{6}{|c|}{ Controle $(\%)-$ B. decumbens } \\
\hline 1 & $97,8 \mathrm{Aa}$ & $99,8 \mathrm{Aa}$ & $98,5 \mathrm{Aa}$ & $100,0 \mathrm{Aa}$ & $98,3 \mathrm{Aa}$ & $100,0 \mathrm{Aa}$ \\
\hline 15 & $93,8 \mathrm{Bb}$ & $100,0 \mathrm{Aa}$ & $90,0 \mathrm{BCb}$ & $100,0 \mathrm{Aa}$ & $93,8 \mathrm{Ab}$ & $100,0 \mathrm{Aa}$ \\
\hline 30 & $96,0 \mathrm{ABb}$ & $99,3 \mathrm{Aa}$ & $92,0 \mathrm{ABb}$ & $99,8 \mathrm{Aa}$ & $95,8 \mathrm{Aa}$ & $99,5 \mathrm{Aa}$ \\
\hline 60 & $85,0 \mathrm{Cb}$ & $99,3 \mathrm{Aa}$ & $85,0 \mathrm{Cb}$ & $99,8 \mathrm{Aa}$ & $84,0 \mathrm{Bb}$ & $98,8 \mathrm{Aa}$ \\
\hline F palha $(\mathbf{P})$ & \multicolumn{2}{|c|}{$81,4 * *$} & \multicolumn{2}{|c|}{$49,45^{* *}$} & \multicolumn{2}{|c|}{$52,7 * *$} \\
\hline F período $(\mathbf{P})$ & \multicolumn{2}{|c|}{$16,70 * *$} & \multicolumn{2}{|c|}{$5,51 * *$} & \multicolumn{2}{|c|}{$13,73 * *$} \\
\hline $\mathbf{F}(\mathbf{P} \times \mathbf{P})$ & \multicolumn{2}{|c|}{$14,88 * *$} & \multicolumn{2}{|c|}{$5,18 * *$} & \multicolumn{2}{|c|}{$9,82 * *$} \\
\hline C.V. (\%) & \multicolumn{2}{|c|}{2,09} & \multicolumn{2}{|c|}{3,58} & \multicolumn{2}{|c|}{2,68} \\
\hline DMS (palha) & \multicolumn{2}{|c|}{2,95} & \multicolumn{2}{|c|}{4,99} & \multicolumn{2}{|c|}{3,77} \\
\hline \multirow[t]{2}{*}{ DMS (período) } & \multicolumn{2}{|c|}{3,94} & \multicolumn{2}{|c|}{6,7} & \multicolumn{2}{|c|}{5,04} \\
\hline & \multicolumn{6}{|c|}{ Controle (\%) - Digitaria sp. } \\
\hline 1 & $99,5 \mathrm{Aa}$ & $100,0 \mathrm{Aa}$ & $99,5 \mathrm{Aa}$ & $100,0 \mathrm{Aa}$ & $99,0 \mathrm{Aa}$ & $100,0 \mathrm{Aa}$ \\
\hline 15 & $98,3 \mathrm{ABa}$ & $100,0 \mathrm{Aa}$ & $94,5 \mathrm{ABa}$ & $100,0 \mathrm{Aa}$ & $94,0 \mathrm{ABa}$ & $100,0 \mathrm{Aa}$ \\
\hline 30 & $94,3 \mathrm{ABb}$ & $100,0 \mathrm{Aa}$ & $81,5 \mathrm{BCb}$ & $100,0 \mathrm{Aa}$ & $80,8 \mathrm{BCb}$ & $100,0 \mathrm{Aa}$ \\
\hline 60 & $94,0 \mathrm{Ba}$ & $97,5 \mathrm{Aa}$ & $73,8 \mathrm{Cb}$ & $98,0 \mathrm{Aa}$ & $73,3 \mathrm{Cb}$ & $95,0 \mathrm{Aa}$ \\
\hline F palha $(\mathbf{P})$ & \multicolumn{2}{|c|}{$8,84 * *$} & \multicolumn{2}{|c|}{$24,70 * *$} & \multicolumn{2}{|c|}{$17,59 * *$} \\
\hline F período $(\mathrm{P})$ & \multicolumn{2}{|c|}{$3,62 *$} & \multicolumn{2}{|c|}{$6,58 * *$} & \multicolumn{2}{|c|}{$5,85 * *$} \\
\hline $\mathbf{F}(\mathbf{P} \times \mathbf{P})$ & \multicolumn{2}{|c|}{$1,38^{\mathrm{ns}}$} & 5,08 & & 3 & \\
\hline C.V. $(\%)$ & 2 , & & 7,4 & & & \\
\hline DMS (palha) & 3 , & & 10 , & & & \\
\hline DMS (período) & 5 , & & 13 , & & & \\
\hline
\end{tabular}

Médias seguidas de mesma letra maiúscula na coluna e minúscula na linha, não diferem estatisticamente entre si pelo teste Tukey $(\mathrm{P}<0,05)$.* - significativo ao nível de $5 \%$ de probabilidade, $* *$ - significativo ao nível de $1 \%$ de probabilidade; ns - não significativo. DAC (dias após a ocorrência de chuvas).

Estes resultados demonstram que uma parcela da dose aplicada chegou ao solo com a chuva simulada de $20 \mathrm{~mm}$, e que a quantidade do produto que chegou ao solo e a associação com a presença de cobertura morta foram bastante eficazes no controle dessa planta daninha. Carbonari et al. (2010) obtiveram resultados semelhantes de controle da mesma espécie após a aplicação da mistura 
formulada de clomazone + hexazinona sobre e sob a palha de cana-de-açúcar.

Para Digitaria sp. (Tabela 1) e I. níveis de controle superiores a $90 \%$ em todos os períodos avaliados para a aplicação sem palha. grandifolia (Tabela 2), foram observados

Tabela 2. Eficácia do flumioxazin no controle de I. nil e I. grandifolia aplicado no solo e na palha de milho, após diferentes períodos sem chuvas. Botucatu/SP - 2010.

\begin{tabular}{|c|c|c|c|c|c|c|}
\hline \multirow[b]{3}{*}{$\begin{array}{l}\text { Períodos } \\
\text { (dias) }\end{array}$} & \multicolumn{6}{|c|}{ Intervalos de avaliação } \\
\hline & \multicolumn{2}{|c|}{$14 \mathrm{DAC}$} & \multicolumn{2}{|c|}{$28 \mathrm{DAC}$} & \multicolumn{2}{|c|}{$42 \mathrm{DAC}$} \\
\hline & Com palha & $\begin{array}{c}\text { Sem } \\
\text { palha }\end{array}$ & $\begin{array}{c}\text { Com } \\
\text { palha } \\
\end{array}$ & $\begin{array}{c}\text { Sem } \\
\text { palha } \\
\end{array}$ & $\begin{array}{c}\text { Com } \\
\text { palha }\end{array}$ & Sem palha \\
\hline & \multicolumn{6}{|c|}{ Controle (\%) - Ipomoea nil } \\
\hline 1 & $77,0 \mathrm{Ab}$ & $98,0 \mathrm{Aa}$ & $87,5 \mathrm{Ab}$ & $97,0 \mathrm{Aa}$ & $88,3 \mathrm{Ab}$ & $97,0 \mathrm{Aa}$ \\
\hline 15 & $77,5 \mathrm{Ab}$ & $95,5 \mathrm{Aa}$ & $86,3 \mathrm{Ab}$ & $94,5 \mathrm{Aa}$ & $88,5 \mathrm{Ab}$ & $95,3 \mathrm{Aa}$ \\
\hline 30 & $57,5 \mathrm{ABb}$ & $80,0 \mathrm{Aa}$ & $76,3 \mathrm{Ba}$ & $82,0 \mathrm{ABa}$ & $79,8 \mathrm{ABa}$ & $79,5 \mathrm{Ba}$ \\
\hline 60 & $56,3 \mathrm{Bb}$ & $77,5 \mathrm{Aa}$ & $74,5 \mathrm{Ba}$ & $80,0 \mathrm{ABa}$ & $78,3 \mathrm{Ba}$ & $82,0 \mathrm{Ba}$ \\
\hline F palha $(\mathbf{P})$ & \multicolumn{2}{|c|}{$28,99 * *$} & \multicolumn{2}{|c|}{$20,92 * *$} & \multicolumn{2}{|c|}{$8,90 * *$} \\
\hline F período $(\mathbf{P})$ & \multicolumn{2}{|c|}{$8,36^{* *}$} & \multicolumn{2}{|c|}{$23,32 * *$} & \multicolumn{2}{|c|}{$20,18 * *$} \\
\hline $\mathbf{F}(\mathbf{P} \times \mathbf{P})$ & \multicolumn{2}{|c|}{$0,06^{\mathrm{ns}}$} & \multicolumn{2}{|c|}{$0,38^{\mathrm{ns}}$} & \multicolumn{2}{|c|}{$1,51^{\mathrm{ns}}$} \\
\hline C.V. $(\%)$ & \multicolumn{2}{|c|}{14,04} & \multicolumn{2}{|c|}{5,29} & \multicolumn{2}{|c|}{5,23} \\
\hline DMS (palha) & \multirow{2}{*}{\multicolumn{2}{|c|}{15,86}} & \multirow{2}{*}{\multicolumn{2}{|c|}{6,54}} & \multirow{2}{*}{\multicolumn{2}{|c|}{6,57}} \\
\hline DMS & & & & & & \\
\hline (período) & \multicolumn{2}{|c|}{21,2} & \multicolumn{2}{|c|}{8,75} & \multicolumn{2}{|c|}{8,78} \\
\hline & \multicolumn{6}{|c|}{ Controle (\%) - Ipomoea grandifolia } \\
\hline 1 & $94,0 \mathrm{Ab}$ & $99,8 \mathrm{Aa}$ & $92,8 \mathrm{Ab}$ & $99,8 \mathrm{Aa}$ & $94,8 \mathrm{Aa}$ & $99,5 \mathrm{Aa}$ \\
\hline 15 & $88,3 \mathrm{Ab}$ & $98,3 \mathrm{Aa}$ & $89,0 \mathrm{ABb}$ & $97,8 \mathrm{Aa}$ & $91,0 \mathrm{Ab}$ & $98,0 \mathrm{ABa}$ \\
\hline 30 & $88,0 \mathrm{Ab}$ & $96,0 \mathrm{Aa}$ & $87,0 \mathrm{ABb}$ & $94,5 \mathrm{Aa}$ & $88,3 \mathrm{ABa}$ & $93,5 \mathrm{ABa}$ \\
\hline 60 & $79,0 \mathrm{Bb}$ & $95,8 \mathrm{Aa}$ & $82,5 \mathrm{Bb}$ & 95,3 Aa & $82,5 \mathrm{Bb}$ & $91,5 \mathrm{Ba}$ \\
\hline F palha $(\mathbf{P})$ & \multicolumn{2}{|c|}{$68,58 * *$} & \multicolumn{2}{|c|}{$50,33 * *$} & \multicolumn{2}{|c|}{$23,75^{* *}$} \\
\hline F período $(P)$ & \multicolumn{2}{|c|}{$10,29 * *$} & \multicolumn{2}{|c|}{$6,40 * *$} & \multicolumn{2}{|c|}{$10,91 * *$} \\
\hline $\mathbf{F}(\mathbf{P} \times \mathbf{P})$ & \multicolumn{2}{|c|}{$3,77 *$} & \multicolumn{2}{|c|}{$1,06^{\mathrm{ns}}$} & & $2^{\text {ns }}$ \\
\hline C.V. (\%) & 3, & & & & & 08 \\
\hline DMS (palha) & 5, & & & & & 51 \\
\hline $\begin{array}{l}\text { DMS } \\
\text { (período) }\end{array}$ & 6? & & & & & 36 \\
\hline
\end{tabular}

Médias seguidas de mesma letra maiúscula na coluna e minúscula na linha, não diferem estatisticamente entre si pelo teste Tukey $(\mathrm{P}<0,05){ }^{*}$ - significativo ao nível de 5\% de probabilidade, ** - significativo ao nível de $1 \%$ de probabilidade; ns - não significativo. DAC (dias após a ocorrência de chuvas)..

Para a aplicação sobre a palha, foram observadas falhas no controle para os períodos de 30 e 60 dias sem ocorrência de chuvas.
Estas perdas podem estar associada a degradação do herbicida na palha, pela ação de microorganismos ou a fotodegradação, uma 
vez que o produto ficou expostos a radiação solar por um longo período na palha. Negrisoli et al. (2009) observaram excelentes níveis de controle (acima de 95\%) de I grandifolia pelo herbicida oxyfluorfen $\left(3,0\right.$ L p.c. ha $\left.{ }^{-1}\right)$ quando aplicado sobre a palha de cana-de-açúcar e submetida à simulação de $30 \mathrm{~mm}$ de chuva, ressaltando-se também a baixa solubilidade deste produto.

Para I. nil (Tabela 2) e B. pilosa (Tabela 3) observou-se também excelentes resultados de controle (acima de 90\%) para os períodos de 1 e 15 dias sem chuva. No entanto, pôde-se observar falhas de controle para os períodos de 30 e 60 dias sem chuva, independente da presença ou ausência de palha. Monquero et al. (2009), avaliando o controle de B. pilosa por herbicidas residuais (trifloxisulfuron + ametrina e metribuzin) aplicados sobre e sob a palha de cana-deaçúcar verificaram que todos os herbicidas utilizados promoveram índices de controle superiores a $90 \%$, em ambas as formas de aplicação.

Para S. rhombifolia (Tabela 3) foram observados excelentes níveis de controle em todos os períodos avaliados para a aplicação do flumioxazin sobre o solo, sem palha. Para a aplicação sobre a palha foram observados níveis inferiores de controle, embora não diferiram estatisticamente da aplicação sem a presença de palha, exceto na primeira avaliação (14 DAA). Carbonari et al. (2008) observaram bons níveis de controle para a mesma espécie até 35 dias após a aplicação do herbicida diclosulam sobre a palha de milheto, seguido de simulação de $30 \mathrm{~mm}$ de chuva.

Nas Tabelas 4, 5 e 6 estão apresentados os resultados de controle das plantas daninhas pelo flumioxazin aplicado na presença e ausência de palha de aveia. Para $B$. decumbens e Digitaria spp (Tabela 4) foram observados excelentes níveis de controle em todos os períodos de avaliação, tanto para a aplicação sobre a palha ou sobre o solo e nos diferentes períodos de permanência do flumioxazin sem a ocorrência de chuva.

Verifica-se bons níveis de controle de I. nil (Tabela 5) quando foi realizada a aplicação do flumioxazin sobre a palha de aveia, independente do período de permanência na palha antes da chuva.Todavia, na aplicação do herbicida sobre o solo, sem a presença de palha, foram observados bons níveis de controle somente quando o produto ficou exposto 1 e 15 dias sem a presença de chuva. A exposição do produto a períodos superiores a 30 dias sem a ocorrência de chuvas, reduziu o nível de controle da espécie.

Para a I. gandifolia foram observados excelentes níveis de controle independente do posicionamento do produto e do período de exposição do mesmo na superfície do solo ou do palha antes da ocorrência da chuva (Tabela 5). Resultado semelhante foi verificado para a especie $S$. rhombifolia (Tabela 6). Carbonari et al. (2008), observaram para aplicação do diclosulam, sob e sobre a palha de sorgo e sobre o solo, excelentes níveis de controle (> 93,5\%) para I. grandifolia e S. rhombifolia, quando o herbicida foi aplicado sobre o solo na ausência de palha de sorgo, sobre o solo com posterior cobertura com a palha e sobre a palha seca e com posterior simulação de 30 mm de chuva.

A espécie B. pilosa foi eficientemente controlada quando foi realizada a aplicação do flumioxazin sobre o solo (na ausência de palha), independentemente do período sem chuva. Para a aplicação sobre a palha de aveia, observa-se excelentes níveis de controle ate 30 dias após a aplicação sem a ocorrência de chuva, com redução da eficiência de controle em período superior (Tabela 6). 
Tabela 3. Eficácia do flumioxazin no controle de B. pilosa e $S$. rhombifolia aplicado no solo e na palha de milho, após diferentes períodos sem chuvas. Botucatu/SP - 2010.

\begin{tabular}{|c|c|c|c|c|c|c|}
\hline \multirow[b]{3}{*}{$\begin{array}{l}\text { Períodos } \\
\text { (dias) }\end{array}$} & \multicolumn{6}{|c|}{ Intervalos de avaliação } \\
\hline & \multicolumn{2}{|c|}{$14 \mathrm{DAC}$} & \multicolumn{2}{|c|}{28 DAC } & \multicolumn{2}{|c|}{42 DAC } \\
\hline & $\begin{array}{l}\text { Com } \\
\text { palha } \\
\end{array}$ & $\begin{array}{c}\text { Sem } \\
\text { palha }\end{array}$ & $\begin{array}{c}\text { Com } \\
\text { palha }\end{array}$ & $\begin{array}{c}\text { Sem } \\
\text { palha }\end{array}$ & Com palha & Sem palha \\
\hline & \multicolumn{6}{|c|}{ Controle (\%) - Bidens pilosa } \\
\hline 1 & $98,5 \mathrm{Aa}$ & $98,8 \mathrm{Aa}$ & $94,5 \mathrm{Aa}$ & $96,3 \mathrm{Aa}$ & $94,0 \mathrm{ABa}$ & $96,3 \mathrm{Aa}$ \\
\hline 15 & $99,0 \mathrm{Aa}$ & $95,5 \mathrm{Aa}$ & $95,8 \mathrm{Aa}$ & $95,0 \mathrm{Aa}$ & $96,3 \mathrm{Aa}$ & $94,8 \mathrm{Aa}$ \\
\hline 30 & $93,3 \mathrm{Aa}$ & $91,3 \mathrm{Aa}$ & $88,3 \mathrm{ABa}$ & $91,8 \mathrm{Aa}$ & $87,0 \mathrm{ABa}$ & $87,0 \mathrm{Aa}$ \\
\hline 60 & 89,3 Aa & $91,8 \mathrm{Aa}$ & $80,0 \mathrm{Bb}$ & $91,3 \mathrm{Aa}$ & $82,0 \mathrm{Ba}$ & $87,5 \mathrm{Aa}$ \\
\hline F palha $(\mathbf{P})$ & \multicolumn{2}{|c|}{$0,15^{\mathrm{ns}}$} & \multicolumn{2}{|c|}{$3,16^{*}$} & \multicolumn{2}{|c|}{$0,44^{\mathrm{ns}}$} \\
\hline $\mathbf{F}$ período $(\mathbf{P})$ & \multicolumn{2}{|c|}{$4,80^{*}$} & \multicolumn{2}{|c|}{$4,53 *$} & \multicolumn{2}{|c|}{$5,52 * *$} \\
\hline $\mathbf{F}(\mathbf{P} \times \mathbf{P})$ & \multicolumn{2}{|c|}{$0,54^{\mathrm{ns}}$} & \multicolumn{2}{|c|}{$1,36^{\mathrm{ns}}$} & \multicolumn{2}{|c|}{$0,42^{\mathrm{ns}}$} \\
\hline C.V. $(\%)$ & \multicolumn{2}{|c|}{5,32} & \multicolumn{2}{|c|}{6,84} & \multicolumn{2}{|c|}{7,34} \\
\hline DMS (palha) & \multicolumn{2}{|c|}{7,34} & \multicolumn{2}{|c|}{9,15} & \multicolumn{2}{|c|}{9,70} \\
\hline \multicolumn{7}{|l|}{ DMS } \\
\hline (período) & \multicolumn{2}{|c|}{9,82} & \multicolumn{2}{|c|}{12,23} & \multicolumn{2}{|c|}{12,98} \\
\hline & \multicolumn{6}{|c|}{ Controle (\%) - Sida rhombifolia } \\
\hline 1 & $100,0 \mathrm{Aa}$ & $100,0 \mathrm{Aa}$ & $100,0 \mathrm{Aa}$ & $100,0 \mathrm{Aa}$ & $100,0 \mathrm{Aa}$ & $100,0 \mathrm{Aa}$ \\
\hline 15 & $97,0 \mathrm{ABa}$ & $100,0 \mathrm{Aa}$ & $96,3 \mathrm{Aa}$ & $100,0 \mathrm{Aa}$ & $98,3 \mathrm{Aa}$ & $100,0 \mathrm{Aa}$ \\
\hline 30 & $94,3 \mathrm{ABa}$ & $99,0 \mathrm{Aa}$ & $94,5 \mathrm{Ab}$ & $100,0 \mathrm{Aa}$ & $94,0 \mathrm{Ab}$ & $100,0 \mathrm{Aa}$ \\
\hline 60 & $93,3 \mathrm{Bb}$ & $100,0 \mathrm{Aa}$ & $95,3 \mathrm{Ab}$ & $100,0 \mathrm{Aa}$ & $94,8 \mathrm{Ab}$ & $100,0 \mathrm{Aa}$ \\
\hline F palha $(\mathbf{P})$ & \multicolumn{2}{|c|}{$9,43 * *$} & \multicolumn{2}{|c|}{$10,48^{* *}$} & \multicolumn{2}{|c|}{$8,2 * *$} \\
\hline F período $(P)$ & \multicolumn{2}{|c|}{$1,92^{\mathrm{ns}}$} & \multicolumn{2}{|c|}{$1,27^{\mathrm{ns}}$} & \multicolumn{2}{|c|}{$1,58^{\mathrm{ns}}$} \\
\hline $\mathbf{F}(\mathbf{P} \times \mathbf{P})$ & 1,4 & & 1,2 & & 1,5 & $8^{\text {ns }}$ \\
\hline C.V. (\%) & 3 & & 3 , & & 3,2 & 26 \\
\hline DMS (palha) & 4 , & & 4 & & $4,($ & 69 \\
\hline DMS & & & & & & \\
\hline (período) & 6 , & & 5 & & 6,2 & 27 \\
\hline
\end{tabular}


Tabela 4. Eficácia do flumioxazin no controle de $B$. decumbens e Digitaria spp aplicado no solo e na palha de milho, após diferentes períodos sem chuvas. Botucatu/SP - 2010.

\begin{tabular}{|c|c|c|c|c|c|c|}
\hline \multirow[b]{3}{*}{ Períodos (dias) } & \multicolumn{6}{|c|}{ Intervalos de avaliação } \\
\hline & \multicolumn{2}{|c|}{14 DAC } & \multicolumn{2}{|c|}{$28 \mathrm{DAC}$} & \multicolumn{2}{|c|}{$42 \mathrm{DAC}$} \\
\hline & Com palha & $\begin{array}{c}\text { Sem } \\
\text { palha }\end{array}$ & $\begin{array}{c}\text { Com } \\
\text { palha }\end{array}$ & $\begin{array}{c}\text { Sem } \\
\text { palha }\end{array}$ & $\begin{array}{l}\text { Com } \\
\text { palha }\end{array}$ & $\begin{array}{c}\text { Sem } \\
\text { palha }\end{array}$ \\
\hline & \multicolumn{6}{|c|}{ Controle (\%) - B. decumbens } \\
\hline 1 & $99,0 \mathrm{Aa}$ & $99,8 \mathrm{Aa}$ & 100,0 & 100,0 & 100,0 & 100,0 \\
\hline 15 & $96,0 \mathrm{ABb}$ & $100,0 \mathrm{Aa}$ & 98,8 & 100,0 & 98,3 & 100,0 \\
\hline 30 & $96,8 \mathrm{ABb}$ & $99,3 \mathrm{Aa}$ & 98,5 & 99,8 & 99,5 & 99,5 \\
\hline 60 & $95,0 \mathrm{Bb}$ & $99,3 \mathrm{Aa}$ & 97,5 & 99,8 & 99,0 & 98,8 \\
\hline F palha $(\mathbf{P})$ & \multicolumn{2}{|c|}{$23,00^{* *}$} & \multicolumn{2}{|c|}{$2,81^{\mathrm{ns}}$} & \multicolumn{2}{|c|}{$1,54^{\mathrm{ns}}$} \\
\hline $\mathbf{F}$ período $(\mathrm{P})$ & \multicolumn{2}{|c|}{$2,41^{*}$} & \multicolumn{2}{|c|}{$0,65^{\mathrm{ns}}$} & \multicolumn{2}{|c|}{$2,63^{\mathrm{ns}}$} \\
\hline $\mathbf{F}(\mathbf{P} \times \mathbf{P})$ & \multicolumn{2}{|c|}{$1,81^{\mathrm{ns}}$} & \multicolumn{2}{|c|}{$0,42^{\mathrm{ns}}$} & \multicolumn{2}{|c|}{$2,34^{\mathrm{ns}}$} \\
\hline C.V. $(\%)$ & \multicolumn{2}{|c|}{1,73} & \multicolumn{2}{|c|}{2,02} & \multicolumn{2}{|c|}{0,86} \\
\hline DMS (palha) & \multicolumn{2}{|c|}{2,47} & \multicolumn{2}{|c|}{2,92} & \multicolumn{2}{|c|}{1,25} \\
\hline DMS (período) & \multicolumn{2}{|c|}{3,31} & \multicolumn{2}{|c|}{3,91} & \multicolumn{2}{|c|}{1,66} \\
\hline & \multicolumn{6}{|c|}{ Controle (\%) - Digitaria sp. } \\
\hline 1 & $98,8 \mathrm{Aa}$ & $100,0 \mathrm{Aa}$ & $100,0 \mathrm{Aa}$ & $100,0 \mathrm{Aa}$ & $99,5 \mathrm{Aa}$ & $100,0 \mathrm{Aa}$ \\
\hline 15 & $96,5 \mathrm{Aa}$ & $100,0 \mathrm{Aa}$ & 99,8 Aa & $100,0 \mathrm{Aa}$ & 99,5 Aa & $100,0 \mathrm{Aa}$ \\
\hline 30 & $93,0 \mathrm{Ab}$ & $100,0 \mathrm{Aa}$ & $99,5 \mathrm{Aa}$ & $100,0 \mathrm{Aa}$ & $99,3 \mathrm{Aa}$ & $100,0 \mathrm{Aa}$ \\
\hline 60 & $94,0 \mathrm{Ab}$ & $97,5 \mathrm{Aa}$ & $97,8 \mathrm{Aa}$ & $98,0 \mathrm{Aa}$ & $97,0 \mathrm{Aa}$ & $95,0 \mathrm{Aa}$ \\
\hline F palha $(\mathbf{P})$ & \multicolumn{2}{|c|}{$12,42 * *$} & \multicolumn{2}{|c|}{$0,31^{\mathrm{ns}}$} & \multicolumn{2}{|c|}{$0,01^{\mathrm{ns}}$} \\
\hline F período $(\mathrm{P})$ & \multicolumn{2}{|c|}{$2,31^{\mathrm{ns}}$} & \multicolumn{2}{|c|}{$5,05^{*}$} & \multicolumn{2}{|c|}{$3,13 *$} \\
\hline $\mathbf{F}(\mathbf{P} \times \mathbf{P})$ & \multicolumn{2}{|c|}{$1,20^{\mathrm{ns}}$} & \multicolumn{2}{|c|}{$0,05^{\mathrm{ns}}$} & & \\
\hline C.V. $(\%)$ & 3,1 & & & & & \\
\hline DMS (palha) & 4,4 & & & & & \\
\hline DMS (período) & 5,9 & & & & & \\
\hline
\end{tabular}

Médias seguidas de mesma letra maiúscula na coluna e minúscula na linha, não diferem estatisticamente entre si pelo teste Tukey $(\mathrm{P}<0,05) . *$ - significativo ao nível de 5\% de probabilidade, ** - significativo ao nível de $1 \%$ de probabilidade; ns - não significativo. DAC (dias após a ocorrência de chuvas). 
Tabela 5. Eficácia do flumioxazin no controle de I. nil e I. grandifolia aplicado no solo e na palha de milho, após diferentes períodos sem chuvas. Botucatu/SP - 2010.

\begin{tabular}{|c|c|c|c|c|c|c|}
\hline & \multicolumn{6}{|c|}{ Intervalos de avaliação } \\
\hline & \multicolumn{2}{|c|}{14 DAC } & \multicolumn{2}{|c|}{28 DAC } & \multicolumn{2}{|c|}{$42 \mathrm{DAC}$} \\
\hline \multirow[t]{2}{*}{ Períodos (dias) } & Com palha & $\begin{array}{c}\text { Sem } \\
\text { palha }\end{array}$ & $\begin{array}{c}\text { Com } \\
\text { palha }\end{array}$ & $\begin{array}{c}\text { Sem } \\
\text { palha }\end{array}$ & $\begin{array}{c}\text { Com } \\
\text { palha }\end{array}$ & $\begin{array}{c}\text { Sem } \\
\text { palha }\end{array}$ \\
\hline & \multicolumn{6}{|c|}{ Controle (\%) - Ipomoea nil } \\
\hline 1 & $86,5 \mathrm{Ab}$ & $98,0 \mathrm{Aa}$ & $91,0 \mathrm{Aa}$ & $97,0 \mathrm{Aa}$ & $92,5 \mathrm{Aa}$ & $97,0 \mathrm{Aa}$ \\
\hline 15 & $86,0 \mathrm{Aa}$ & $95,5 \mathrm{Aa}$ & $88,8 \mathrm{Aa}$ & $94,5 \mathrm{Aa}$ & $89,5 \mathrm{Aa}$ & 95,3 Aa \\
\hline 30 & $86,5 \mathrm{Aa}$ & $80,0 \mathrm{Ba}$ & $87,5 \mathrm{Aa}$ & $82,0 \mathrm{Ba}$ & $85,5 \mathrm{Aa}$ & $79,5 \mathrm{Ba}$ \\
\hline 60 & $80,8 \mathrm{Aa}$ & $77,5 \mathrm{Ba}$ & $86,0 \mathrm{Aa}$ & $80,0 \mathrm{Ba}$ & $87,5 \mathrm{Aa}$ & $82,0 \mathrm{Ba}$ \\
\hline F palha $(\mathbf{P})$ & \multicolumn{2}{|c|}{$1,41^{\mathrm{ns}}$} & \multicolumn{2}{|c|}{$0,01^{\mathrm{ns}}$} & \multicolumn{2}{|c|}{$0,03^{\mathrm{ns}}$} \\
\hline F período $(\mathrm{P})$ & \multicolumn{2}{|c|}{$6,87 * *$} & \multicolumn{2}{|c|}{$8,33 * *$} & \multicolumn{2}{|c|}{$11,74 * *$} \\
\hline $\mathbf{F}(\mathbf{P} \times \mathbf{P})$ & \multicolumn{2}{|c|}{$3,61^{*}$} & \multicolumn{2}{|c|}{$3,35^{*}$} & \multicolumn{2}{|c|}{$3,36^{*}$} \\
\hline C.V. $(\%)$ & \multicolumn{2}{|c|}{7,77} & \multicolumn{2}{|c|}{5,88} & \multicolumn{2}{|c|}{5,49} \\
\hline DMS (palha) & \multicolumn{2}{|c|}{9,80} & \multicolumn{2}{|c|}{7,61} & \multicolumn{2}{|c|}{7,10} \\
\hline \multirow[t]{2}{*}{ DMS (período) } & \multicolumn{2}{|c|}{13,10} & \multicolumn{2}{|c|}{10,13} & \multicolumn{2}{|c|}{9,49} \\
\hline & \multicolumn{6}{|c|}{ Controle (\%) - Ipomoea grandifolia } \\
\hline 1 & $95,3 \mathrm{Aa}$ & $99,8 \mathrm{Aa}$ & $94,3 \mathrm{Ab}$ & $99,8 \mathrm{Aa}$ & $94,8 \mathrm{Aa}$ & $99,5 \mathrm{Aa}$ \\
\hline 15 & $94,0 \mathrm{Aa}$ & $98,3 \mathrm{Aa}$ & $95,8 \mathrm{Aa}$ & $97,8 \mathrm{Aa}$ & $94,8 \mathrm{Aa}$ & $98,0 \mathrm{ABa}$ \\
\hline 30 & $92,5 \mathrm{Aa}$ & $96,0 \mathrm{Aa}$ & $93,3 \mathrm{Aa}$ & $94,5 \mathrm{Aa}$ & 93,3 Aa & $93,5 \mathrm{ABa}$ \\
\hline 60 & $91,0 \mathrm{Ab}$ & $95,8 \mathrm{Aa}$ & $91,8 \mathrm{Aa}$ & $95,3 \mathrm{Aa}$ & $91,3 \mathrm{Aa}$ & $91,5 \mathrm{Ba}$ \\
\hline F palha $(\mathbf{P})$ & \multicolumn{2}{|c|}{$14,57 * *$} & \multicolumn{2}{|c|}{$5,77^{*}$} & \multicolumn{2}{|c|}{$2,22^{\mathrm{ns}}$} \\
\hline F período $(\mathbf{P})$ & \multicolumn{2}{|c|}{$2,77 *$} & \multicolumn{2}{|c|}{$2,10^{\mathrm{ns}}$} & \multicolumn{2}{|c|}{$3,52 *$} \\
\hline $\mathbf{F}(\mathbf{P} \times \mathbf{P})$ & \multicolumn{2}{|c|}{$0,06^{\mathrm{ns}}$} & & & & \\
\hline C.V. $(\%)$ & 3,3 & & & & & \\
\hline DMS (palha) & 4,6 & & & & & \\
\hline DMS (período) & 6,1 & & & & & \\
\hline
\end{tabular}

Médias seguidas de mesma letra maiúscula na coluna e minúscula na linha, não diferem estatisticamente entre si pelo teste Tukey $(\mathrm{P}<0,05) . *$ - significativo ao nível de 5\% de probabilidade, ** - significativo ao nível de $1 \%$ de probabilidade; ns - não significativo. DAC (dias após a ocorrência de chuvas). 
Tabela 6. Eficácia do flumioxazin no controle de B. pilosa e $S$. rhombifolia aplicado no solo e na palha de milho, após diferentes períodos sem chuvas. Botucatu/SP - 2010.

\begin{tabular}{|c|c|c|c|c|c|c|}
\hline \multirow[b]{3}{*}{$\begin{array}{c}\text { Períodos } \\
\text { (dias) }\end{array}$} & \multicolumn{6}{|c|}{ Intervalos de avaliação } \\
\hline & \multicolumn{2}{|c|}{14 DAC } & \multicolumn{2}{|c|}{28 DAC } & \multicolumn{2}{|c|}{$42 \mathrm{DAC}$} \\
\hline & Com palha & $\begin{array}{c}\text { Sem } \\
\text { palha }\end{array}$ & $\begin{array}{c}\text { Com } \\
\text { palha }\end{array}$ & $\begin{array}{c}\text { Sem } \\
\text { palha }\end{array}$ & $\begin{array}{c}\text { Com } \\
\text { palha }\end{array}$ & Sem palha \\
\hline & \multicolumn{6}{|c|}{ Controle (\%) - Bidens pilosa } \\
\hline 1 & $97,0 \mathrm{Aa}$ & $98,8 \mathrm{Aa}$ & $96,5 \mathrm{Aa}$ & $96,3 \mathrm{Aa}$ & 94,8 & 96,3 \\
\hline 15 & $98,5 \mathrm{Aa}$ & $95,5 \mathrm{Aa}$ & $96,8 \mathrm{Aa}$ & $95,0 \mathrm{Aa}$ & 94,0 & 94,8 \\
\hline 30 & $98,0 \mathrm{Aa}$ & $91,3 \mathrm{Aa}$ & $96,3 \mathrm{Aa}$ & $91,8 \mathrm{Aa}$ & 94,0 & 87,0 \\
\hline 60 & $86,5 \mathrm{Ba}$ & $91,8 \mathrm{Aa}$ & $85,0 \mathrm{Bb}$ & $91,3 \mathrm{Aa}$ & 88,3 & 87,5 \\
\hline F palha $(\mathbf{P})$ & \multicolumn{2}{|c|}{$0,16^{\mathrm{ns}}$} & \multicolumn{2}{|c|}{$0,01^{\mathrm{ns}}$} & \multicolumn{2}{|c|}{$0,47^{\mathrm{ns}}$} \\
\hline F período $(\mathbf{P})$ & \multicolumn{2}{|c|}{$5,30 *$} & \multicolumn{2}{|c|}{$6,70^{*}$} & \multicolumn{2}{|c|}{$3,09^{\mathrm{ns}}$} \\
\hline $\mathbf{F}(\mathbf{P} \times \mathbf{P})$ & \multicolumn{2}{|c|}{$2,40^{\mathrm{ns}}$} & \multicolumn{2}{|c|}{$2,43^{\mathrm{ns}}$} & \multicolumn{2}{|c|}{$0,93^{\mathrm{ns}}$} \\
\hline C.V. $(\%)$ & \multicolumn{2}{|c|}{5,11} & \multicolumn{2}{|c|}{4,42} & \multicolumn{2}{|c|}{6,15} \\
\hline DMS (palha) & \multirow{2}{*}{\multicolumn{2}{|c|}{7,05}} & \multirow{2}{*}{\multicolumn{2}{|c|}{6,04}} & \multirow{2}{*}{\multicolumn{2}{|c|}{8,27}} \\
\hline DMS & & & & & & \\
\hline (período) & \multicolumn{2}{|c|}{9,44} & \multicolumn{2}{|c|}{8,07} & \multicolumn{2}{|c|}{11,05} \\
\hline & \multicolumn{6}{|c|}{ Controle (\%) - Sida rhombifolia } \\
\hline 1 & 100,0 & 100,0 & 100,0 & 100,0 & 100,0 & 100,0 \\
\hline 15 & 99,8 & 100,0 & 100,0 & 100,0 & 99,3 & 100,0 \\
\hline 30 & 100,0 & 99,0 & 100,0 & 100,0 & 99,8 & 100,0 \\
\hline 60 & 100,0 & 100,0 & 100,0 & 100,0 & 99,3 & 100,0 \\
\hline F palha $(\mathbf{P})$ & \multicolumn{2}{|c|}{$0,53^{\mathrm{ns}}$} & \multicolumn{2}{|c|}{ - } & \multicolumn{2}{|c|}{$3,58^{\mathrm{ns}}$} \\
\hline F período $(\mathbf{P})$ & \multicolumn{2}{|c|}{$0,84^{\mathrm{ns}}$} & \multicolumn{2}{|c|}{ - } & \multicolumn{2}{|c|}{$0,66^{\mathrm{ns}}$} \\
\hline $\mathbf{F}(\mathbf{P} \times \mathbf{P})$ & \multicolumn{2}{|c|}{$1,16^{\mathrm{ns}}$} & & & & $6^{\text {ns }}$ \\
\hline C.V. $(\%)$ & 0,7 & & & & & 65 \\
\hline DMS (palha) & 1,0 & & & & & 95 \\
\hline DMS & & & & & & \\
\hline (período) & 1,4 & & & & & 28 \\
\hline
\end{tabular}

Médias seguidas de mesma letra maiúscula na coluna e minúscula na linha, não diferem estatisticamente entre si pelo teste Tukey $(\mathrm{P}<0,05){ }^{*}$ - significativo ao nível de 5\% de probabilidade, $* *$ - significativo ao nível de $1 \%$ de probabilidade; ns - não significativo. DAC (dias após a ocorrência de chuvas).

\section{Conclusões}

De maneira geral, pode-se observar que a aplicação do flumioxazin sobre o solo ou sobre a palha de milho e aveia promoveu bons níveis de controle das plantas daninhas. Pode-se observar também uma tendência de redução nos níveis de controle para algumas espécies de plantas daninhas, quando o produto foi exposto a períodos acima de 30 dias entre a aplicação e ocorrência de chuvas, indicando que houve degradação do produto quando submetido a períodos extensos de exposição na superfície da palha ou do solo sem a ocorrência de chuva. 


\section{Referências}

CARBONARI, C. A. et al. Efeitos de períodos de permanência de clomazone + hexazinona no solo e na palha de cana-de-açúcar antes da ocorrência de chuvas na eficácia de controle de plantas daninhas. Planta Daninha, v.28, n.1, p.197-205, 2010.

CARBONARI, C.A. et al. Eficácia do herbicida diclosulam em associação com a palha de sorgo no controle de Ipomoea grandifolia e Sida rhombifolia. Planta Daninha, v.26, n.3, p.657-664, 2008.

FIDELIS, R.R. et al. Alguns aspectos do plantio direto para a cultura da soja. Bioscience Journal, v.19, n.1, p23-31, 2003.

FORNAROLLI, D.A. et al. Influência da cobertura morta no comportamento do herbicida atrazine. Planta Daninha, v.16, n.2, p.97-107, 1998.

GOMES JR, F.G.; CHRISTOFFOLETI, P.J. Biologia e manejo de plantas daninhas em áreas de plantio direto. Planta Daninha, v.26, n.4, p.789-798, 2008.

LOCKE, M.A.; BRYSON, C.T. Herbicide-soil interaction in reduced tillage and plant residue management systems. Weed Science, v.45, n.2, p.307-320, 1997.

MACIEL, C.D.G.; VELINI, E.D. Simulação do caminhamento da água da chuva e herbicidas em palhadas utilizadas em sistema de plantio direto. Planta Daninha, v.23, n.3, p.471-482, 2005.

MIYAZAWA, M.; PAVAN, M.A.; FRANCHINI, J. C. Neutralização da acidez do perfil do solo por resíduos vegetais. Informações Agronômicas, v.92, n.1, p.1-8, 2000.

MONQUERO, P.A. et al. Eficácia de herbicidas aplicados em diferentes épocas sobre B. pilosa e I. quamoclit em área de cana- de-açúcar colhida mecanicamente. Planta Daninha, v.27, n.3, p.563-570, 2009.

MORAES, P.V.D. et al. Manejo de plantas de cobertura no controle de plantas daninhas na cultura do milho. Planta Daninha, v.27, n.2, p.289-296, 2009.

NEGRISOLI, E. Eficácia do herbicida oxyfluorfen com a cobertura de palha no controle de plantas daninhas. Planta Daninha, v.27, n.1, p.197-203, 2009.

OLIVEIRA, M. F.; SILVA, A. A.; NEVES, J. C. L. Influência do tamanho do agregado e do nível de umidade do solo na atividade do flumioxazin. Revista Ceres, v.45, n.257, p.8187, 1998.

PAES, J.M.V.; REZENDE, A.M. Manejo de plantas daninhas no sistema plantio direto na palha. Informe Agropecuário, v.22, n.208, p.37-42, 2001.

RODRIGUES, B.N. Influência da cobertura morta no comportamento dos herbicidas imazaquin e clomazone. Planta Daninha, v.11, n.1/2, p.21-8, 1993.

RODRIGUES, B. N.; ALMEIDA, F. S. Guia de herbicidas. 5 ed. Londrina: Edição dos Autores, 2005.

ROSSI, C.V.S. Dinâmica e eficácia no controle de plantas daninhas pelo herbicida metribuzin aplicado sobre palha de canade-açúcar. 2004. 95 f. Dissertação (Mestrado em Agronomia/Proteção de Plantas) Universidade Estadual Paulista, Botucatu, 2004.

SOCIEDADE BRASILEIRA DA CIÊNCIA DAS PLANTAS DANINHAS. Procedimentos para instalação, avaliação e análise de experimentos com herbicidas. Londrina: SBCPD, 1995. 42 p.

TOFOLI, G.R. et al. Dinâmica do tebuthiuron em palha de cana-de-açúcar. Planta Daninha, v.27, n.4, p.815-821, 2009. 